\title{
On Euler products with smaller than one exponents
}

\author{
Gábor Román \\ Eötvös Loránd University, Hungary \\ email: romangabor@caesar.elte.hu
}

\begin{abstract}
Investigation has been made regarding the properties of the $\prod_{p<n}\left(1 \pm 1 / p^{s}\right)$ products over the prime numbers, where we fix the $s \in \mathbb{R}$ exponent, and let the $n \geq 2$ natural bound grow toward positive infinity. The nature of these products for the $s \geq 1$ case is known. We get approximations for the case when $s \in[1 / 2,1)$, furthermore different observations for the case when $s<1 / 2$.
\end{abstract}

\section{Introduction}

In this article, we will investigate the asymptotical properties of Euler products. More precisely, we are going to look at how does the

$$
\prod_{p \leq n}\left(1 \pm \frac{1}{p^{s}}\right)
$$

products over the prime numbers behave asymptotically, when we fix the $s \in \mathbb{R}$ exponent, and let the $\mathrm{n} \geq 2$ natural bound grow toward positive infinity.

Due to the connection with the Riemann zeta function and Dirichlet series, the "classical" Euler products were and are the subject of a thorough investigation. Some of the results concerning them can describe the nature of the products which we will examine, so we are going to shortly sum up the properties which can be already stated based on these results.

2010 Mathematics Subject Classification: 11M99

Key words and phrases: Euler product, Mertens' theorem, Riemann zeta function, Abel summation, Riemann hypothesis, prime number theorem, logarithmic integral 
First we concentrate on the negative case of expression (1). It is well known that

$$
\lim _{n \rightarrow \infty} \prod_{p \leq n}\left(1-\frac{1}{p^{s}}\right)=\frac{1}{\zeta(s)}
$$

when $s>1$, see [2] section 11.5. (This result dates back to Euler, who pointed out this connection in [9] by using simple algebraic manipulations on - what is now known as - the Dirichlet series form of the Riemann zeta function.) As when $s=1$, Mertens proved his infamous result in [12], stating that

$$
\lim _{n \rightarrow \infty} \ln n \prod_{p \leq n}\left(1-\frac{1}{p}\right)=e^{-\gamma}
$$

holds, which is usually referred to as Mertens' third theorem. (To obtain this result, one has to know how the $\zeta(\mathrm{s})$ Riemann zeta function behaves in the neighbourhood of its pole at $s=1$.) When $s=0$, then the product is zero, and when $s$ is negative, then the product diverges.

Concerning the positive case of expression (1), one can use the

$$
\left(1+\frac{1}{p^{s}}\right)\left(1-\frac{1}{p^{s}}\right)=1-\frac{1}{p^{2 s}}
$$

equation when $s \in \mathbb{R}$, to transform the results from the negative case. Because of this, we get that

$$
\lim _{n \rightarrow \infty} \prod_{p \leq n}\left(1+\frac{1}{p^{s}}\right)=\frac{\zeta(s)}{\zeta(2 s)}
$$

when $s>1$, and also that

$$
\lim _{n \rightarrow \infty} \frac{1}{\ln n} \prod_{p \leq n}\left(1+\frac{1}{p}\right)=\frac{6}{\pi^{2}} e^{\gamma}
$$

holds when $s=1$. (These results are not new, see again section 11.5 in [2].) When $s$ is zero, the product grows as $2^{\pi(n)}$, where $\pi$ is the prime counting function.

What remains in both cases is when $s \in(0,1)$, and $s<0$ in the positive case of expression (1). We are going to concentrate on the positive case, because equation (2) can be applied to transform our results back to the negative case. We will rely on the following theorem, see theorem 2.7.1 from [3]. 
Theorem 1 Let $\mathrm{f}$ be continuously differentiable on an open interval containing $[2, \infty)$, and let $\pi(x)=\operatorname{Li}(x)+\epsilon(x)$, where $\operatorname{Li}(x)=\int_{2}^{x} d t / \ln (t)$ is the offset logarithmic integral. Now if $\mathrm{x} \geq 2$, then

$$
\sum_{p \leq x} f(p)=\int_{2}^{x} \frac{f(t)}{\ln (t)} d t+\epsilon(x) f(x)-\int_{2}^{x} \epsilon(t) f^{\prime}(t) d t .
$$

The precision which we can achieve while applying this theorem depends heavily on the applied $\epsilon$ error term. Most of the results in this area give absolute bound to the $\pi(x)-\operatorname{li}(x)=\varepsilon(x)$ error term, where

$$
\operatorname{li}(x)=\lim _{h \rightarrow 0^{+}}\left(\int_{0}^{1-h} \frac{d t}{\ln t}+\int_{1+h}^{x} \frac{d t}{\ln t}\right)
$$

is the logarithmic integral. Take note that the applied $\epsilon$ and the later $\varepsilon$ differs; we are going to use the later $\varepsilon$ throughout the paper. Because of this, we have to substitute the $\epsilon$ error term in theorem 1 as

$$
\epsilon(x)=\pi(x)-\operatorname{Li}(x)=\pi(x)-\operatorname{li}(x)+\operatorname{li}(2)=\varepsilon(x)+\operatorname{li}(2)
$$

later on. Take note that the value of $\operatorname{li}(2)$ is 1.04516378 approximately. Regarding the $\varepsilon$ error term, Koch showed in [10], that if the Riemann hypothesis is true, then $\varepsilon(x) \in \mathcal{O}(\sqrt{x} \ln x)$. This has been made more precise by Schoenfeld in [14], showing that

$$
|\mathcal{E}(x)|<\frac{\sqrt{x} \ln x}{8 \pi}
$$

holds for all $x \geq 2657$. As of now, this is the best possible error bound depending on the validity of the Riemann hypothesis. A weaker result from the same article of Koch states that

$$
|\varepsilon(x)|<\mathcal{O}\left(x^{1 / 2+\sigma}\right)
$$

for all $\sigma>0$, if the Riemann hypothesis is true. Kotnik in his [11] article improves this by conjecturing that even

$$
|\varepsilon(x)|<\sqrt{x}
$$

holds for all $x \geq 2$ according to his investigations. We will use this later inequality in our calculations. Now we give our results for the $s \in[1 / 2,1)$ case. 
Proposition 1 If the conjecture of Kotnik is true, then

$$
\lim _{n \rightarrow \infty} e^{-l i\left(n^{1-s}\right)} \prod_{p \leq n}\left(1+\frac{1}{p^{s}}\right)=\varphi(s) e^{\mathcal{O}\left(\Gamma\left(1-\frac{1}{2 s}\right)\right)}
$$

holds when $s \in(1 / 2,1)$, where

$$
\varphi(s)=\frac{\sqrt{2 s-1}}{(1-s) \sqrt{\ln 2}}
$$

furthermore

$$
\prod_{p \leq n}\left(1+\frac{1}{\sqrt{p}}\right)=e^{\mathrm{li}(\sqrt{n})+\mathcal{O}(\ln n)}
$$

holds when $\mathrm{s}=1 / 2$.

In the case when Kotnik's conjecture would turn out to be false, one could fall back to using the result of Schoenfeld, see inequality (3), which would yield similar results, but with a more complex right hand side in equation (5), furthermore a much rougher asymptotic in place of equation (7). The plot of the $\varphi(s)$ function for $s \in(1 / 2,1)$ can be seen on figure 1 .

Take note that the gamma function on the right hand side of equation (5) goes to positive infinity as $s$ approaches $1 / 2$ from the right, and $\varphi(s)$ goes to positive infinity as $s$ approaches 1 from the left. When $s$ is not near $1 / 2$ or 1 , the right hand side of equation (5) is smaller than a constant depending on s, because next to the $\Gamma$ function, we only have constant terms hidden behind the asymptotic, see the proof in section 2 .

It is noted that to obtain the third theorem of Mertens, one has to know how the Riemann zeta function behaves in the neighbourhood of its pole. Here, the Riemann hypothesis is a much stronger assumption, which relies on the exact behaviour of the Riemann zeta function in the critical strip. (For more information about the critical strip, see for example [7].)

As for the cases when $s \in(0,1 / 2)$ and when $s<0$, we are going to get much rougher results. These will be more like observations, and we are going to give them in section 3 , where we will state our remarks. 


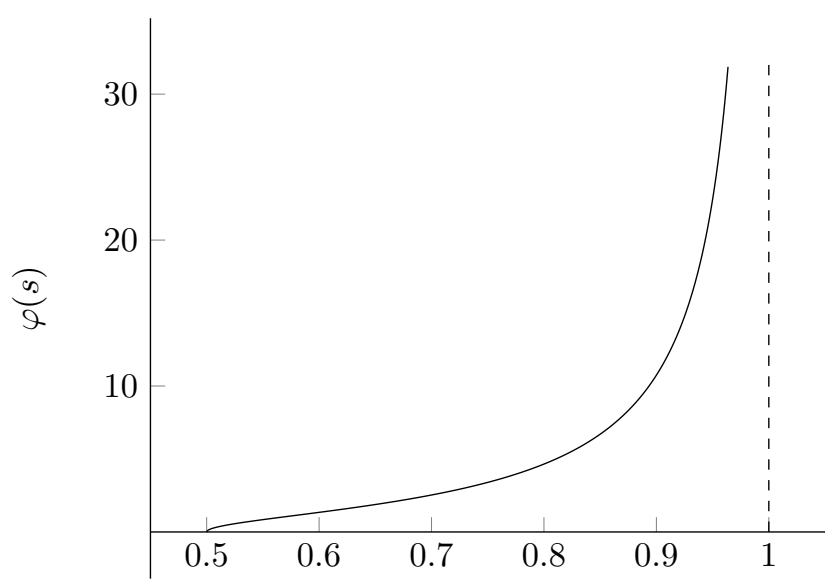

$s$

Figure 1: Plot of the $\varphi(s)$ function for $s \in(1 / 2,1)$ from equation (6). Take note that $\varphi(s)$ approaches positive infinity as $s$ approaches 1 from the left.

Our results have strong connection with the logarithmic integral, which is not so surprising due to the fact that we apply theorem 1 to obtain them. We know, see either chapter 5, equation 5.1.3 and 5.1.10 in [1], or article [6], that

$$
\operatorname{li}(x)=\gamma+\ln \ln x+\sum_{k=1}^{\infty} \frac{\ln ^{k} x}{k ! k}
$$

holds when $x>1$. As one approaches $x=1$ either from the left or the right, the li $(x)$ logarithmic integral grows toward negative infinity. Using similar arguments as in [6], one can derive a formula, which is very similar to equation $(8)$, in the case when $x \in(0,1)$.

Lemma 1 When $x \in(0,1)$, then

$$
\operatorname{li}(x)=\gamma+\ln \ln \frac{1}{x}+\sum_{k=1}^{\infty} \frac{\ln ^{k} x}{k ! k}
$$

holds.

Proof. Let $x \in(0,1)$. By substituting $t$ with $e^{-u}$, we get that

$$
\operatorname{li}(x)=\int_{0}^{x} \frac{1}{\ln t} d t=-\int_{-\ln x}^{\infty} \frac{e^{-\mathfrak{u}}}{u} d u=-\int_{0}^{\infty} \frac{e^{-u}}{u} d u+\int_{0}^{-\ln x} \frac{e^{-u}}{u} d u
$$


equalities hold, because $-\ln x>0$ as $x \in(0,1)$. By splitting the first integral on the right hand side, we get

$$
-\int_{0}^{1} \frac{e^{-u}}{u} d u-\int_{1}^{\infty} \frac{e^{-u}}{u} d u+\int_{0}^{-\ln x} \frac{e^{-u}}{u} d u+\int_{\ln x}^{-\ln x} \frac{1}{u} d u
$$

where one should observe that the last term which we have added is zero. We want to introduce $\gamma$ into this expression, and because

$$
\gamma=\int_{0}^{1} \frac{1-e^{-u}}{u} d u-\int_{1}^{\infty} \frac{e^{-u}}{u} d u
$$

holds, see page 103 of [5], we want to "cut down" $\int_{0}^{1} 1 / u d u$ from the last term in expression (9). Two scenarios can occur based on the value of $x$. If $x \in\left(0, e^{-1}\right)$, then $-\ln x \geq 1$, so one can do the

$$
\int_{\ln x}^{-\ln x} \frac{1}{u} d u=\int_{\ln x}^{0} \frac{1}{u} d u+\int_{0}^{1} \frac{1}{u} d u+\int_{1}^{-\ln x} \frac{1}{u} d u
$$

split, from which we get that expression (9) is equal to

$$
\gamma+\ln \ln \frac{1}{x}+\int_{0}^{-\ln x} \frac{e^{-u}}{u} d u+\int_{\ln x}^{0} \frac{1}{u} d u
$$

where interchanging the limits of the integration in the third term, and by applying the $\boldsymbol{u}=-\mathrm{t}$ substitution in the last term, one can get

$$
\gamma+\ln \ln \frac{1}{x}+\int_{-\ln x}^{0} \frac{1-e^{-u}}{u} d u
$$

where the integral can be exchanged with the sum given in the lemma, as in article [6]. If $x \in\left(e^{-1}, 1\right)$, then $0<-\ln x<1$, so one can do the

$$
\int_{\ln x}^{-\ln x} \frac{1}{u} d u=\int_{\ln x}^{0} \frac{1}{u} d u+\int_{0}^{1} \frac{1}{u} d u-\int_{-\ln x}^{1} \frac{1}{u} d u
$$

split, which we can transform back to the previous case by interchanging the limits of the integration in the last term.

Take note that when $x \in(0,1)$, then $\operatorname{li}(x)$ is smaller than zero and monotone decreases toward negative infinity, furthermore when $x \in(1,+\infty)$ then it monotone increases from negative infinity to positive infinity, see figure 2.

The reason why we have given the results in proposition 1 by using the logarithmic integral, and not dissecting it further is because it is hard to give a concise and also precise approximation for the logarithmic integral with elementary functions. 


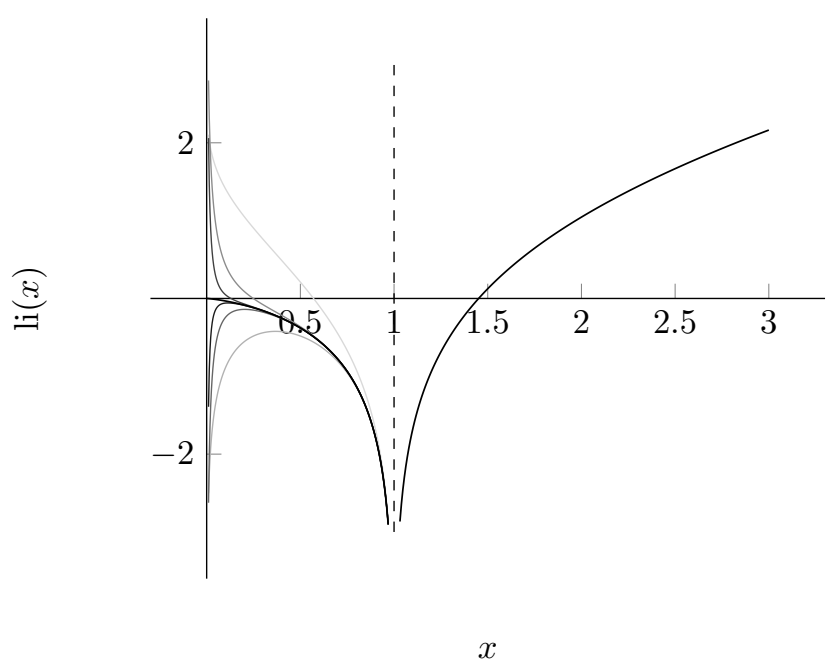

Figure 2: Plot of the logarithmic integral. The curves between 0 and 1 correspond to the truncated versions of the equation from lemma 1. (They get darker as we take more terms from the sum.)

\section{Proof of the proposition}

As it is noted, from now on we are going to concentrate on the positive case of expression (1), while $s \in[1 / 2,1)$ holds.

Proof. Changing the product into summation in expression (1), we get

$$
\exp \left(\ln \prod_{p \leq n}\left(1+\frac{1}{p^{s}}\right)\right)=\exp \left(\sum_{p \leq n} \ln \left(1+\frac{1}{p^{s}}\right)\right)
$$

where we are going to apply theorem 1 on the argument of the exponential function on the right hand side. According theorem 1 and our observations after it, this sum is equal to

$$
\int_{2}^{n} \frac{\ln \left(1+\frac{1}{t^{s}}\right)}{\ln t} d t+(\varepsilon(n)+\operatorname{li}(2)) \ln \left(1+\frac{1}{n^{s}}\right)+s \int_{2}^{n} \frac{\varepsilon(t)+\operatorname{li}(2)}{t\left(t^{s}+1\right)} d t
$$

because there exists an open interval containing $[2, \infty)$ on which we can continuously differentiate $\ln \left(1+1 / t^{s}\right)$, furthermore

$$
\frac{d}{d t} \ln \left(1+\frac{1}{t^{s}}\right)=-\frac{s}{t\left(t^{s}+1\right)}
$$


holds in the said interval. In the following sections we will examine the terms of expression (11) piecewise, then sum our results in section 2.4.

\section{1 $\quad$ First term}

Concerning the first term in expression (11), we are going to use a series representation of the logarithmic function from [1], equation 4.1.24, which goes as

$$
\ln (1+x)=x-\frac{1}{2} x^{2}+\frac{1}{3} x^{3}+\ldots=\sum_{k=1}^{\infty} \frac{(-1)^{k+1} x^{k}}{k}
$$

where $|x| \leq 1$ and $x \neq-1$. So

$$
\int_{2}^{n} \frac{\ln \left(1+\frac{1}{t^{s}}\right)}{\ln t} d t=\int_{2}^{n} \sum_{k=1}^{\infty} \frac{(-1)^{k+1}}{k t^{k s} \ln t} d t=\int_{2}^{n} \sum_{k=1}^{\infty} f_{k, s}(t) d t
$$

because $\left|1 / t^{s}\right| \leq 1$ when $t \in[2, n]$ and $s \in[1 / 2,1]$. Now we are going to show that the order of the integration and the summation can be interchanged in equation (13). For every $k>0$ and $s \in[1 / 2,1]$, we have that $f_{k, s}$ is continuous on $[2, n]$, which means that it is measurable on $[2, n]$. What we have to show is that

$$
\sum_{k=1}^{\infty} \int_{2}^{n}\left|f_{k, s}(t)\right| d t=\sum_{k=1}^{\infty} \int_{2}^{n} \frac{1}{k t^{k s} \ln t} d t<\frac{1}{\ln 2} \sum_{k=1}^{\infty} \int_{2}^{n} \frac{1}{t^{k s}} d t
$$

converges. Because the integrand on the right hand side is a positive, measurable function on $[2, n]$ for every $k>0$ and $s \in[1 / 2,1]$, we can interchange the order of the summation and the integration, which - based on the sum of the geometric series - gives us

$$
\frac{1}{\ln 2} \int_{2}^{n} \sum_{k=1}^{\infty} \frac{1}{t^{k s}} d t=\frac{1}{\ln 2} \int_{2}^{n} \frac{1}{t^{s}-1} d t \leq \frac{1}{\ln 2} \int_{2}^{n} \frac{1}{\sqrt{t}-1} d t<+\infty
$$

because $s \in[1 / 2,1]$, so one can interchange the order of summation and integration in equation (13) as

$$
\int_{2}^{n} \sum_{k=1}^{\infty} \frac{(-1)^{k+1}}{k t^{k s} \ln t} d t=\sum_{k=1}^{\infty} \frac{(-1)^{k+1}}{k} \int_{2}^{n} \frac{1}{t^{k s} \ln t} d t
$$

where we have two cases during the evaluation of the integral inside the summation. 
1. If $s=1 / m$ for some $m>0$ integer, then the integral on the right side in equation (14) will be simply $\ln \ln n-\ln \ln 2$ when $k=m$.

2. Otherwise, when $s \in(1 / 2,1)$ and there doesn't exist an $m$ integer such that $s=1 / \mathrm{m}$, then the integral can be treated as follows. Let us set $\mathrm{t}=\mathrm{r}^{\lambda}$, then we get that

$$
\int_{2}^{n} \frac{1}{t^{k s} \ln t} d t=\int_{2^{1 / \lambda}}^{n^{1 / \lambda}} \frac{r^{\lambda-k s \lambda-1}}{\ln r} d r
$$

where we want $\lambda-k s \lambda-1$ to be zero, so we have to set $\lambda=1 /(1-k s)$, which gives us that

$$
\int_{2^{1 / \lambda}}^{n^{1 / \lambda}} \frac{r^{\lambda-k s \lambda-1}}{\ln r} d r=\int_{2^{1-k s}}^{n^{1-k s}} \frac{1}{\ln r} d r=\operatorname{li}\left(n^{1-k s}\right)-\operatorname{li}\left(2^{1-k s}\right)
$$

holds in this case.

Using these results, we get that when $s=1 / m$ for some $m>0$ integer, then equation (14) is equal to

$$
\frac{(-1)^{m+1}}{m}(\ln \ln n-\ln \ln 2)+\sum_{k \in \mathbb{N}^{+} \backslash\{m\}} \frac{(-1)^{k+1}}{k}\left(\operatorname{li}\left(n^{1-k s}\right)-\operatorname{li}\left(2^{1-k s}\right)\right)
$$

otherwise when $s \in(1 / 2,1)$ and there doesn't exist an $m$ integer such that $s=1 / \mathrm{m}$, then equation (14) is equal to the

$$
\sum_{k=1}^{\infty} \frac{(-1)^{k+1}}{k}\left(\operatorname{li}\left(n^{1-k s}\right)-\operatorname{li}\left(2^{1-k s}\right)\right)
$$

sum. We are going to investigate these sums depending on the value of $s$ separately in the cases when $s \in(1 / 2,1)$, and when $s=1 / 2$.

\subsubsection{Above half}

When $s \in(1 / 2,1)$, then there is surely no such $m$ integer that $s=1 / m$, so we are going to concentrate on expression (16) in this case. We will show that the sum can be actually split into two sums; one which contains only the li $\left(\mathrm{n}^{1-\mathrm{ks}}\right)$ terms, and another, which contains only the $\operatorname{li}\left(2^{1-k s}\right)$ terms. Considering the members of the first sum, when $1-\mathrm{ks}<0$, then

$$
\lim _{n \rightarrow \infty} \operatorname{li}\left(n^{1-k s}\right)=0
$$


holds. Because $s \in(1 / 2,1)$, this is true when $k>1$. Regarding the members of the second sum, because the logarithmic integral is negative on $(0,1)$, we have that

$$
0<\frac{-\operatorname{li}\left(2^{1-\mathrm{ks}}\right)}{\mathrm{k}}=-\frac{1}{\mathrm{k}} \lim _{\mathrm{h} \rightarrow 0^{+}} \int_{\mathrm{h}}^{2^{1-\mathrm{ks}}} \frac{1}{\ln \mathrm{t}} \mathrm{dt}
$$

holds for $k>2$ and $s \in(1 / 2,1)$. With these assumptions about $k$ and $s$, we have that the $1 / \ln t$ function is continuous on $\left[h, 2^{1-k s}\right]$ for every $h \in\left(0,2^{1-k s}\right)$, so we can apply the mean value theorem and get that the right hand side of equation (17) is smaller than

$$
-\frac{1}{k} \lim _{h \rightarrow 0^{+}} \frac{2^{1-k s}-h}{\ln 2^{1-k s}}=\frac{2^{1-k s}}{k(k s-1) \ln 2}
$$

because $|\ln t|$ increases as $t$ approaches zero from the right. Take note that the right hand side of equation (18) decreases monotonically to zero as $k$ approaches infinity when $s \in(1 / 2,1)$. To simplify the discussion, we introduce the

$$
\alpha_{i, s}(x):=\sum_{k=i}^{\infty} \frac{(-1)^{k+1}}{k} \operatorname{li}\left(x^{1-k s}\right)
$$

notation. We have arrived at the conclusion that $\alpha_{2, s}(n)$ converges to zero as $n$ approaches positive infinity, and $\alpha_{3, s}(2)$ also converges based on the alternating series test, so the sum in expression (16) can be split, and it is equal to

$$
\operatorname{li}\left(n^{1-s}\right)+\alpha_{2, s}(n)-\operatorname{li}\left(2^{1-s}\right)+\frac{1}{2} \operatorname{li}\left(2^{1-2 s}\right)+\mathcal{O}(1)
$$

when $s \in(1 / 2,1)$. Now we are going to bound the third and fourth terms in this last expression. Using equation (8) for the third term, we have

$$
\operatorname{li}\left(2^{1-s}\right)=\gamma+\ln \ln 2^{1-s}+\mathcal{O}(1)
$$

furthermore using the result of lemma 1 for the fourth term, we get

$$
\operatorname{li}\left(2^{1-2 s}\right)=\gamma+\ln \ln \frac{1}{2^{1-2 s}}+\mathcal{O}(1)
$$

where the

$$
\sum_{k=1}^{\infty} \frac{\ln ^{k} x}{k ! k}<\sum_{k=0}^{\infty} \frac{\ln ^{k} x}{k !}=x
$$


inequality was applied in both cases. Summing these results, we have that expression (16) is equal to

$$
\operatorname{li}\left(n^{1-s}\right)+\alpha_{2, s}(n)+\ln \frac{\sqrt{2 s-1}}{(1-s) \sqrt{\ln 2}}+\mathcal{O}(1)
$$

in this case. Now we keep the $\alpha_{2, s}(n)$ term, because it will disappear when we will let $n$ approach positive infinity at the end.

\subsubsection{At half}

As we lower $s$ from one toward zero, the $s=1 / 2$ is the first case where we have to use expression (15), which gives us

$$
\operatorname{li}(\sqrt{n})-\operatorname{li}(\sqrt{2})-\frac{1}{2}(\ln \ln n-\ln \ln 2)+\sum_{k=3}^{\infty} \frac{(-1)^{k+1}}{k}\left(\operatorname{li}\left(n^{1-k / 2}\right)-\operatorname{li}\left(2^{1-k / 2}\right)\right)
$$

where we can split the sum again, based on the arguments in section 2.1.1. Summing the constants, we get that expression (15) is equal to

$$
\operatorname{li}(\sqrt{\mathfrak{n}})+\alpha_{3,1 / 2}(\mathfrak{n})-\frac{1}{2} \ln \ln n+\mathcal{O}(1)
$$

when $s=1 / 2$. Yet again, the $\alpha_{3,1 / 2}(n)$ term will disappear when we will approach positive infinity with $n$ at the end.

\subsection{Second term}

As for the second term in expression (11), we are going to use the inequalities 4.1.33 from [1], which state that

$$
\frac{x}{1+x}<\ln (1+x)<x
$$

holds for every $x>-1, x \neq 0$, from which it follows that

$$
\frac{1}{x^{s}+1}<\ln \left(1+\frac{1}{x^{s}}\right)<\frac{1}{x^{s}}
$$

holds for every $x \in(0, \infty)$ and $s \in \mathbb{R}$. Using the error term from inequality (4) in the second term of expression (11), we get that

$$
\left|(\varepsilon(n)+\operatorname{li}(2)) \ln \left(1+\frac{1}{n^{s}}\right)\right| \leq(|\varepsilon(n)|+\operatorname{li}(2)) \ln \left(1+\frac{1}{n^{s}}\right)
$$




$$
<\frac{\sqrt{n}+\operatorname{li}(2)}{n^{s}}
$$

so if $s>1 / 2$, then the absolute value of the second term converges to zero as $n$ goes to infinity. When $s=1 / 2$, then its absolute value is smaller than

$$
1+\frac{\operatorname{li}(2)}{\sqrt{n}}
$$

otherwise, its absolute value behaves asymptotically as

$$
\mathcal{O}\left(n^{1 / 2-s}\right)
$$

when $s<1 / 2$.

\subsection{Third term}

For the third term in expression (11), we can assume without loss of generality that $\varepsilon$ and also $|\varepsilon|$ is Riemann-integrable on $[2, n]$, so we can write

$$
\left|s \int_{2}^{n} \frac{\varepsilon(t)+l i(2)}{t\left(t^{s}+1\right)} d t\right| \leq s \int_{2}^{n} \frac{|\varepsilon(t)|+\operatorname{li}(2)}{t\left(t^{s}+1\right)} d t<s \int_{2}^{n} \frac{\sqrt{t}+l i(2)}{t\left(t^{s}+1\right)} d t
$$

where we have substituted the error term from inequality (4). This is equal to

$$
\beta_{s}(n):=2 s\left[\sqrt{t} \cdot{ }_{2} F_{1}\left(1, \frac{1}{2 s} ; 1+\frac{1}{2 s} ;-t^{s}\right)\right]_{2}^{n}+\operatorname{li}(2)\left[\ln \frac{t^{s}}{t^{s}+1}\right]_{2}^{n}
$$

because the first part of the integral on the right hand side can be transformed into the form of the ${ }_{2} \mathrm{~F}_{1}$ Gauss hypergeometric function, and the second part can be decomposed into partial fractions. The transformation of the first part can be done as

$$
\begin{aligned}
\int \frac{1}{\sqrt{x}\left(x^{s}+1\right)} d x & =\int_{0}^{x} u^{-\frac{1}{2}}\left(1+u^{s}\right)^{-1} d u+C \\
& =\frac{1}{s} \int_{0}^{x^{s}} r^{\frac{1}{2 s}-1}(1+r)^{-1} d r+C \\
& =\frac{\sqrt{x}}{s} \int_{0}^{1} t^{\frac{1}{2 s}-1}\left(1+x^{s} t\right)^{-1} d t+C
\end{aligned}
$$

where first we switched the indefinite integral into a definite one, then we have applied the $u^{s}=r$ substitution and finally the $r=x^{s} t$ substitution. The Gauss hypergeometric function can be written in the

$$
{ }_{2} F_{1}(a, b ; c ; z)=\frac{\Gamma(c)}{\Gamma(b) \Gamma(c-b)} \int_{0}^{1} t^{b-1}(1-t)^{c-b-1}(1-t z)^{-a} d t
$$


form, see equation 15.3.1 in [1]. Using this, we have that $z=-\chi^{s}, a=1$ and $\mathrm{b}=1 / 2 \mathrm{~s}$. Because $\mathrm{c}-\mathrm{b}-1=0$ should hold, we get our missing $\mathrm{c}$ and with it, the first part of expression (25). As for the second part, we have

$$
\operatorname{li}(2) \int_{2}^{n} \frac{s}{t\left(t^{s}+1\right)} d t=\operatorname{li}(2) \int_{2}^{n} \frac{s}{t}-\frac{s t^{s-1}}{t^{s}+1} d t
$$

where the second fraction is a logarithmic derivative. We are going to investigate the resulting expression (25) separately in the cases when $s \in(1 / 2,1)$, and when $s=1 / 2$.

\subsubsection{Above half}

First, we deal with the upper limit of the integration. Substituting $n$ into expression (25), we get

$$
2 s \sqrt{n} \cdot{ }_{2} F_{1}\left(1, \frac{1}{2 s} ; 1+\frac{1}{2 s} ;-n^{s}\right)+\operatorname{li}(2) \ln \frac{n^{s}}{n^{s}+1}
$$

which, by using the

$$
{ }_{2} \mathrm{~F}_{1}(\mathrm{a}, \mathrm{b} ; \mathrm{c} ; z)=(1-z)^{-\mathrm{b}}{ }_{2} \mathrm{~F}_{1}\left(\mathrm{~b}, \mathrm{c}-\mathrm{a} ; \mathrm{c} ; \frac{z}{z-1}\right)
$$

linear transformation formula, see equation 15.3.5 in [1], can be transformed into the

$$
2 s \frac{\sqrt{n}}{\left(n^{s}+1\right)^{1 / 2 s}} 2 F_{1}\left(\frac{1}{2 s}, \frac{1}{2 s} ; 1+\frac{1}{2 s} ; \frac{n^{s}}{n^{s}+1}\right)+\operatorname{li}(2) \ln \frac{n^{s}}{n^{s}+1}
$$

form. Because $s \in(1 / 2,1)$, we get that

$$
\lim _{n \rightarrow+\infty} \frac{\sqrt{n}}{\left(n^{s}+1\right)^{1 / 2 s}}{ }_{2} F_{1}\left(\frac{1}{2 s}, \frac{1}{2 s} ; 1+\frac{1}{2 s} ; \frac{n^{s}}{n^{s}+1}\right)={ }_{2} F_{1}\left(\frac{1}{2 s}, \frac{1}{2 s} ; 1+\frac{1}{2 s} ; 1\right)
$$

holds. When $c$ is not zero or a negative integer, furthermore $\mathfrak{R}(c-a-b)>0$ is true, then one can apply the

$$
{ }_{2} \mathrm{~F}_{1}(\mathrm{a}, \mathrm{b} ; \mathrm{c} ; 1)=\frac{\Gamma(\mathrm{c}) \Gamma(\mathrm{c}-\mathrm{a}-\mathrm{b})}{\Gamma(\mathrm{c}-\mathrm{a}) \Gamma(\mathrm{c}-\mathrm{b})}
$$

substitution, see equation 15.1.20 in [1]. Because $s \in(1 / 2,1)$, the conditions are satisfied, and we can utilise the above mentioned formula to get

$$
2 s_{2} F_{1}\left(\frac{1}{2 s}, \frac{1}{2 s} ; 1+\frac{1}{2 s} ; 1\right)=2 s \Gamma\left(1+\frac{1}{2 s}\right) \Gamma\left(1-\frac{1}{2 s}\right)
$$


which has an anomaly at $s=1 / 2$. For the second term, observe that

$$
\lim _{n \rightarrow+\infty} \ln \frac{n^{s}}{n^{s}+1}=0
$$

holds. Now we deal with the lower limit of the integral. By substituting 2 into expression (25) we get

$$
2 s \sqrt{2} \cdot{ }_{2} F_{1}\left(1, \frac{1}{2 s} ; 1+\frac{1}{2 s} ;-2^{s}\right)+\operatorname{li}(2) \ln \frac{2^{s}}{2^{s}+1}
$$

where the second term is a small constant, so we will concentrate on the value of the hypergeometric function. Using another

$$
{ }_{2} \mathrm{~F}_{1}(\mathrm{a}, \mathrm{b} ; \mathrm{c} ; z)=(1-z)^{-\mathrm{a}}{ }_{2} \mathrm{~F}_{1}\left(\mathrm{a}, \mathrm{c}-\mathrm{b} ; \mathrm{c} ; \frac{z}{z-1}\right)
$$

linear transformation formula, see equation 15.3 .4 in [1], we get that the hypergeometric function in expression (28) is equal to

$$
\frac{1}{2^{s}+1} 2^{2} F_{1}\left(1,1 ; 1+\frac{1}{2 s} ; \frac{2^{s}}{2^{s}+1}\right)
$$

where the last argument is in $(0,1)$. We are going to give an upper bound for this expression. If $\mathrm{a} \leq 1$ and $0<\mathrm{b} \leq \mathrm{c}$, then

$$
{ }_{2} \mathrm{~F}_{1}(-\mathrm{a}, \mathrm{b} ; \mathrm{c} ; z)^{1 / \mathrm{a}} \geq\left[\left(1-\frac{\mathrm{b}}{\mathrm{c}}\right)+\frac{\mathrm{b}}{\mathrm{c}}(1-z)^{\mathrm{a}}\right]^{1 / \mathrm{a}}
$$

for all $z \in(0,1)$, see [8] furthermore [13] and [4]. Because $s \in(1 / 2,1)$, we can apply this inequality, which means that

$$
{ }_{2} \mathrm{~F}_{1}\left(1,1 ; 1+\frac{1}{2 s} ; \frac{2^{\mathrm{s}}}{2^{\mathrm{s}}+1}\right) \leq\left(1-\frac{1}{1+\frac{1}{2 s}}\right)+\frac{1}{1+\frac{1}{2 s}}\left(1-\frac{2^{s}}{2^{s}+1}\right)^{-1}
$$

which is just a small constant when $s \in(1 / 2,1)$. Joining our results so far, we get that $\beta_{s}(\mathfrak{n})$ from expression (25) then converges to the right hand side of equation (27) plus some constant as $n$ approaches positive infinity when $s \in(1 / 2,1)$. 


\subsubsection{At half}

One of the special elementary cases of the hypergeometric function is the

$$
{ }_{2} \mathrm{~F}_{1}(1,1 ; 2 ; z)=-z^{-1} \ln (1-z)
$$

equality, see equation 15.1.3 in [1]. This means that when $s=1 / 2$, then the first part of expression (25) is equal to

$$
[\ln (\sqrt{t}+1)]_{2}^{n} \in \mathcal{O}(\ln n)
$$

and because we have tried to bound the absolute value of the third term from above, this partial result already spoils our pursuit of reaching a constant error term in this special case. Regarding the second part of expression (25), what was said in the previous section still holds, meaning that the second part converges to a small constant as $\mathrm{n}$ approaches infinty.

\subsection{Summing the parts}

Now we are going to sum our results. In the case when $s \in(1 / 2,1)$, the first term of expression (11) is equal to expression (19), the absolute value of the second term is smaller than the right hand side of inequality (21) and the absolute value of the third term is smaller than expression (25). So expression (11) is equal to

$$
\operatorname{li}\left(n^{1-s}\right)+\ln \frac{\sqrt{2 s-1}}{(1-s) \sqrt{\ln 2}}+\mathcal{O}(1)+\mathcal{O}\left(\alpha_{2, s}(n)+\frac{\sqrt{n}+\operatorname{li}(2)}{n^{s}}+\beta_{s}(n)\right)
$$

in this case. Reintroducing this into equation (10), we get that the positive case of expression (1) is equal to

$$
\frac{\sqrt{2 s-1}}{(1-s) \sqrt{\ln 2}} \exp \left(\operatorname{li}\left(n^{1-s}\right)+\mathcal{O}(1)+\mathcal{O}\left(\alpha_{2, s}(n)+\frac{\sqrt{n}+\operatorname{li}(2)}{n^{s}}+\beta_{s}(n)\right)\right)
$$

where, after dividing with $\exp \left(\operatorname{li}\left(n^{1-s}\right)\right)$ and taking the limit in $n$, we get the sought equality (5). As for the case when $s=1 / 2$ the first term of expression (11) is equal to expression (20), the absolute value of the second term is smaller than expression (22), and the absolute value of the third term is smaller than expression (29). By these, expression (11) is equal to

$$
\operatorname{li}(\sqrt{n})+\mathcal{O}(\ln n)
$$

and by reintroducing this into equation (10), we get equality (7). 


\section{Remarks}

\subsection{Below half}

The problem in this region is that either when using expression (15) or expression (16) the result becomes more and more complicated as we approach zero with $s$ from the right. This happens mainly because the li $\left(n^{1-k s}\right)$ terms only disappear when $k>1 / s$, but also because one has to pay close attention to the li $\left(2^{1-k s}\right)$ terms when $2^{1-k s}$ is close to one. (The li $\left(n^{1-k s}\right)$ terms behave more nicely, because they avoid the anomaly at one.) Furthermore, without taking the third term into consideration, the second term already contributes a rough asymptotical term, see expression (23).

\subsection{The case of negative exponents}

When the s exponent is negative, instead of expression (11), the sum in equation (10) is equal to

$$
\int_{2}^{n} \frac{\ln \left(1+t^{|s|}\right)}{\ln t} d t+(\varepsilon(n)+\operatorname{li}(2)) \ln \left(1+n^{|s|}\right)-|s| \int_{2}^{n} \frac{\varepsilon(t)+\operatorname{li}(2)}{t^{1-|s|}\left(1+t^{|s|}\right)} d t
$$

because there exists an open interval containing $[2, \infty)$ on which we can continuously differentiate $\ln \left(1+t^{|s|}\right)$, furthermore

$$
\frac{d}{d t} \ln \left(1+t^{|s|}\right)=|s| \frac{t^{|s|-1}}{1+t^{|s|}}
$$

holds. We cannot apply equation (12) like in section 2.1. Instead, we will use the following simple estimations, which can be shown using the properties of the logarithmic function. For every $s>0$ real number, there exists such $c_{s}>1$ constant, that

$$
\ln x^{s} \leq \ln \left(1+x^{s}\right) \leq c_{s} \ln x^{s}
$$

holds for every $x>1$ real number. Applying these estimations on the first term on expression (30), we get

$$
|s| \int_{2}^{n} \mathrm{dt} \leq \int_{2}^{n} \frac{\ln \left(1+\mathrm{t}^{|s|}\right)}{\ln \mathrm{t}} \mathrm{dt} \leq \mathrm{c}_{\mathrm{s}}|\mathrm{s}| \int_{2}^{\mathrm{n}} \mathrm{dt}
$$

which shows us that the first term grows as $\Theta(n)$. Proceeding to the second term in expression (30), we have that its absolute value is smaller than

$$
(|\mathcal{E}(n)|+\operatorname{li}(2)) \ln \left(1+n^{|s|}\right) \leq c_{s}|s|(\sqrt{n}+\operatorname{li}(2)) \ln n
$$


which grows as $\mathcal{O}(\sqrt{n} \ln n)$. Still assuming that $\varepsilon$ and also $|\varepsilon|$ is Riemannintegrable on $[2, n]$, the absolute value of the third term in expression (30) is smaller than or equal to

$$
|s| \int_{2}^{n} \frac{|\varepsilon(t)|+\operatorname{li}(2)}{t^{1-|s|}\left(1+t^{|s|}\right)} d t
$$

which we can estimate from above by dropping the plus one from the denominator. This way, we get

$$
|s| \int_{2}^{n} \frac{|\varepsilon(t)|+\operatorname{li}(2)}{t} d t<|s| \int_{2}^{n} \frac{\sqrt{t}+\operatorname{li}(2)}{t} d t=|s|[2 \sqrt{t}+\operatorname{li}(2) \ln t]_{2}^{n}
$$

which grows as $\mathcal{O}(\sqrt{n})$. As it can be seen, when the s exponent is negative in equation (10), then the first term dominates so the positive case of the product in expression (1) grows as $\exp (\Theta(n))$.

\subsection{Remark about the strength of the method}

Finally, we are going to look at how the theorem performs in the $s=1$ case. Following the same path as in section 2, for the first term in expression (11) we should use expression (15), which is equal to

$$
\ln \ln n-\ln \ln 2+\sum_{k=2}^{\infty} \frac{(-1)^{k+1}}{k}\left(\operatorname{li}\left(n^{1-k}\right)-\operatorname{li}\left(2^{1-k}\right)\right)
$$

in this case. As in section 2.1.1, we can deduce that this expression is equal to

$$
\ln \ln n+\alpha_{2,1}(n)+\mathcal{O}(1)
$$

where the $\alpha_{2,1}(n)$ term disappears as we approach positive infinity with $n$. The absolute value of the second term in expression (11) is smaller than

$$
\frac{\sqrt{n}+\operatorname{li}(2)}{n}
$$

which vanishes as $\mathrm{n}$ tends to positive infinity, so what remains is the absolute value of the third term in the expression (11). Based on inequality (24) we get that its absolute value is smaller than

$$
\beta(n):=\int_{2}^{n} \frac{\sqrt{t}+\operatorname{li}(2)}{t(t+1)} d t=2[\arctan \sqrt{t}]_{2}^{n}+\left[\ln \frac{t}{t+1}\right]_{2}^{n}
$$


which converges to a small constant when $\mathrm{n}$ approaches infinity. Using expression (31), expression (32), and finally expression (33), we get that equation (10) is equal to

$$
\exp \left(\ln \ln n+\mathcal{O}(1)+\mathcal{O}\left(\alpha_{2,1}(n)+\frac{\sqrt{n}+\operatorname{li}(2)}{n}+\beta(n)\right)\right)
$$

which in turn means that

$$
\lim _{n \rightarrow \infty} \frac{1}{\ln n} \prod_{p \leq n}\left(1+\frac{1}{p}\right)=e^{\mathcal{O}(1)}
$$

holds.

\section{Acknowledgments}

The author wishes to thank the reviewer for the helpful remarks.

\section{References}

[1] M. Abramowitz, I. A. Stegun, Handbook of Mathematical Functions with Formulas, Graphs, and Mathematical Tables, Applied Mathematics Series 55, tenth printing, Dover publications, (1972).

[2] T. M. Apostol Introduction to Analytic Number Theory, Undergraduate Texts in Mathematics, Springer-Verlag, (1976).

[3] E. Bach, J. Shallit, Algorithmic Number Theory, Volume 1: Efficient Algorithms, The MIT Press, (1996).

[4] R. W., Barnard, K. C. Richards, A Note on the Hypergeometric Mean Value, Comput. Meth. Funct. Th., 1 (1) (2001), 81-88.

[5] B. C. Berndt, Ramanujan's Notebooks, Part I., Springer, (1985).

[6] B. C. Berndt, R. J. Evans, Some elegant approximations and asymptotic formulas of Ramanujan, J. Comput. Appl. Math., 37 (1991), 35-41.

[7] R. P. Brent, On the Zeros of the Riemann Zeta Function in the Critical Strip. Math. Comp., 33 (148) (1979), 1361-1372. 
[8] B. C. Carlson, Some inequalities for hypergeometric functions. Proc. Amer. Math. Soc., 17 (1966), 32-39.

[9] L. Euler, Variae observationes circa series infinitas. Commentarii academiae scientiarum Petropolitanae, 9 (1737), 160-188.

[10] H. Koch, Sur la distribution des nombres premiers. Acta Math., 24 (1901), 159-182.

[11] T. Kotnik, The prime-counting function and its analytic approximations, $\pi(x)$ and its approximations. Adv. Comput. Math., 29 (1) (2008), 55-70.

[12] F. Mertens, Ein Beitrag zur analytischen Zahlentheorie, J. reine angew. Math., 78 (1874), 46-62.

[13] K. C. Richards, Sharp power mean bounds for the Gaussian hypergeometric function. J. Math. Anal. Appl., 308 (1) (2005), 303-313.

[14] L. Schoenfeld, Sharper Bounds for the Chebyshev Functions $\theta(x)$ and $\psi(x)$. II. Math. Comp., 30 (134) (1976), 337-360.

Received: August 15, 2019 